\title{
Research Methods Associated with Situational Simulations in Construction
}

\author{
Amlan Mukherjee, ${ }^{1}$ Nilufer Onder ${ }^{2}$ and Eddy M. Rojas ${ }^{3}$ \\ ${ }^{1}$ Assistant Professor, Department of Civil and Environmental Engineering, Michigan \\ Tech, Houghton, MI 49931; email: amukherj@mtu.edu \\ ${ }^{2}$ Associate Professor, Department of Computer Science, Michigan Tech, Houghton, \\ MI 49931; email: nilufer@mtu.edu \\ ${ }^{3}$ Associate Professor, Department of Construction Management, University of \\ Washington, Seattle, WA 98195; email: er@u.washington.edu
}

\begin{abstract}
:
Situational simulations create temporally dynamic clinical exercises of construction project scenarios that expose participants to rapidly unfolding events and the pressures of decision-making. They further construction research and education, by providing an interactive simulation platform that can be used to explore "what-if" construction scenarios, estimate risks and contingencies, test alternative plans during construction, and support the capture and analysis of real-time project data to support expert decision-making. The design, development, and use of situational simulations calls upon an inter-disciplinary approach which includes methodologies that are rooted in modeling of discrete event systems, intelligent planning, statistical data mining and cognitive psychology. We trace the development of two related situational simulation systems, the Virtual Coach and the Interactive Construction Decision-Making Aid (ICDMA) that have been developed by research groups at University of Washington and Michigan Tech. with technical support from industry. Over the last five years, the simulations have significantly evolved in their abilities to represent and reason about construction projects, as tools that enhance construction education and as experimental test beds that can be used to study construction decision-making. The research efforts have resulted in different aspects of this research to be funded by two funding agencies.
\end{abstract}

\section{Introduction}

Situational simulations are dynamic, interactive, context-sensitive, adaptive environments. They simulate construction project scenarios in keeping with rules that govern the construction management $(\mathrm{CM})$ domain in general, and the project being simulated in particular. In such an environment, participants are exposed to diverse project management scenarios and situations that rapidly unfold in time. They can react to these scenarios by making strategic decisions involving resource and activity 
scheduling, with the goal of completing the project at hand within schedule and under budget. The simulation allows them to explore "what-if" scenarios to test implications of their decisions in a safe environment, without the fear of incurring any real losses. In brief, construction situational simulations are akin to first-person construction manager strategy games. The participants of situational simulations come from both academia and industry, carrying diverse goals including education of construction managers as well as the interactive planning and management of construction projects.

The goals of developing CM situational simulations are: (i) provide undergraduate students with realistic, interactive learning environments that integrate related concepts, (ii) create interactive contingency planners that can be used to manage complex construction projects, and (iii) formally study dynamic decisionmaking under uncertainty in CM crisis scenarios. This research is motivated by the growing demand for qualified construction managers, and the need to improve planning in $\mathrm{CM}$ using advances in computing and simulations.

The genesis of situational simulations happened at the University of Washington. The first version was called Virtual Coach - a simulation of a simple eight-activity hypothetical construction project. The implementation had two goals: (i) illustrate the ability of developed models to represent and reason about CM information in a general-purpose simulation platform and (ii) illustrate the ability of situational simulations to improve construction management education. The next version, the Interactive Construction Decision-Making Aid (ICDMA), was implemented at Michigan Tech. using an improved constraint network representation and reasoning method. It simulated the construction of a real four-storey steel framed office building. The goals of this implementation were: (i) illustrate the general ability to simulate real construction projects and (ii) illustrate the use of situational simulations for construction planning. Currently a third version is being developed in a collaborative effort between University of Washington and Michigan Tech. This implementation combines the advances made with Virtual Coach and ICDMA, and also provides the ability to formally capture and analyze human decision-making data using the simulation. The goals of this implementation are: (i) rigorously deploy the simulation across multiple construction management classrooms in partner schools to assess undergraduate educational outcomes of using complex situational simulations, (ii) gain insight into decision-making in dynamic uncertain environments using situational simulation environments as experimental test beds to gather and analyze human decision-making data, and (iii) develop and test a significantly improved interface capable of efficiently visualizing construction management information.

The design, development, and use of situational simulations calls upon an inter-disciplinary approach which includes methodologies that are rooted in modeling of discrete event systems, intelligent planning, statistical data mining, and education. In addition, it requires a commitment from the construction industry to participate in experiments and provide access to construction project data. In this paper, we discuss ongoing and completed research in situational simulations, with a focus on the methodological questions that have been addressed in the process of modeling the $\mathrm{CM}$ domain, implementing and testing the interactive simulations as platforms for research and education. The paper is divided into four parts. The first part places this 
research within the context of $\mathrm{CM}$ simulations and explains the underlying conceptual foundations. The second part defines the formal mathematical models driving the simulation. The third part explains the pragmatics of the design and implementation of situational simulation to generate general-purpose construction simulation environments. The final part discusses current and future applications of situational simulations in studying $\mathrm{CM}$ planning, $\mathrm{CM}$ decision-making, and furthering construction education.

\section{Conceptual Foundation of Situational Simulations}

Construction simulations can be classified using different approaches. One approach is whether they are simulating CM processes or construction operations. Simphony (Hajjar and AbouRizk 1999) and STROBOSCOPE (Martinez and Ioannou 1999) are examples of simulations that deal with construction operations such as tunneling and earthmoving. The second approach is based on whether they are of a special purpose or a general purpose in nature. Special purpose simulations are restricted in scope to a particular operation like tunneling or a particular management process like bidding, while general-purpose simulations allow for greater flexibility of scope since they are programmable. A survey of current research indicates that the general focus of simulation research as been in developing general purpose and special purpose simulation of construction operations (STROBOSCOPE and Simphony). There are few efforts at developing general-purpose simulation platforms of CM processes. This research has moved towards the development of a general-purpose framework (GPF) for an interactive situational simulation platform for CM processes.

Rojas and Mukherjee (2006) discuss the conceptual foundations of the GPF based on a classification of the preconstruction and construction phase processes into specific classes of problems. During the preconstruction phase, the problem at hand is that of creating a resource-loaded activity schedule, also referred to as the "AsPlanned" schedule. This is classified as a constraint satisfaction problem (CSP). During the construction phase, managers face the challenge of completing the project while dynamically making critical decisions that satisfy the constraints encoded in the As-Planned schedule by reallocating resources, rescheduling activities, and making time-cost trade-offs. Hence, the manager's job during the construction phase is akin to a contingency planning problem In summary, the conceptual foundation of situational simulations is based on the abstraction of the CM domain to a planning problem during the project implementation phase, and a constraint satisfaction problem during the pre-construction phase. In the next section we discuss the development of a formal approach used to develop and implement the GPF that reflects the conceptual foundations discussed in this section.

\section{Formal Foundation of Situational Simulations}

The GPF is built using a "language" that represents and reasons about activities, actions, and events in the CM domain. The semantics of such a general representation are used to represent and reason about scenarios in the CM domain as a combination of constraint satisfaction problem within a dynamic partial-observability planning system. In addition, situational simulations use an underlying mathematical model that is used to update the productivity and cost in the simulated construction project as it progresses from simulation day to day. 
Representation and Reasoning Model: Based on the axioms of time, using First Order Logic, a formal language was developed that allowed expressive representation and reasoning of CM information (Rojas and Mukherjee 2005a). The entire simulation environment is represented as a set of variables that take values from continuous or discrete ranges. Each such variable is attached to an interval of time, which specifies the interval over which the value of the variable is valid. A change in the status of one or more variables represents a situation when an event has occurred. This temporal representation of the construction environment as a combination of variables is used to reason about complex construction scenarios.

For example, consider the narrative: In the event of a labor strike of duration denoted by the time interval $t$, productivity for all activities is reduced to 0 due to a $0 \%$ availability of labor. Formally, we represent productivity by the variable prod and labor by the variable labor. In the event of a labor strike the variables are set to the null state for the duration of the strike denoted by the time interval - $t$. This is represented across all activity contexts by the variable set: \{labor(null, $t)$, prod(null, $t)\}$. Assuming there was $100 \%$ productivity for a time interval of $t$ ' before the strike, the situation before the strike started is represented by: $\left\{\operatorname{labor}\left(100 \%, t^{\prime}\right), \operatorname{prod}(100 \%\right.$, $\left.\left.t^{\prime}\right)\right\}$ where the predicate $\operatorname{Meets}\left(t^{\prime}, t\right)$ asserts that the time interval is $t^{\prime}$ immediately before $t$. This event represents a violation in a resource constraint, i.e., labor, which is a necessary resource, is missing. This can be logically asserted by the following:

$$
\begin{aligned}
\forall t \cdot A c t \text { Labor_Strike }(\text { t.start }) \Rightarrow & \exists t^{\prime} \cdot \operatorname{labor}\left(100 \%, t^{\prime}\right) \wedge \operatorname{prod}\left(100 \%, t^{\prime}\right) \\
& \wedge \operatorname{Event}(\text { Labor_Strike, } t) \wedge \operatorname{ImmBefore}\left(t^{\prime}, t\right)
\end{aligned}
$$

A detailed description of the representation and reasoning can be found in (Rojas and Mukherjee 2005).

Events are represented as an assertion of the pre-conditions that lead to its occurrence and post-conditions that describe the impact and duration of the event. Actions are immediate inputs that trigger the change in the states of the variables in the pre-condition set to reflect the post-conditions for the duration of the event. The action represents a simulation triggered random event (weather), the impact of a user decision (such as reduced productivity on site resulting in congestion). An event $E$ is represented by triples $<E_{\text {prec }}, E_{\text {eff, }} E_{\text {prob }}>$ where $E_{\text {prec }}$, and $E_{\text {eff }}$ are sets of variables and $E_{\text {prob }}$ is the event probability. Hence, in the previous example, the action Act_Labor_Strike creates the event Labor_Strike that belongs to the set of events $\boldsymbol{E}$. The associated variable sets defining it are: $E_{\text {prec }}=\left\{\operatorname{labor}\left(100 \%, t^{\prime}\right), \operatorname{prod}\left(100 \%, t^{\prime}\right)\right\}$ and $E_{\text {eff }}=\{l a b o r($ null, $t), \operatorname{prod}($ null, $t)\}$. The triples are defined in a knowledgebase that drives the simulation and allows it to identify a violation that can cause the event. In this way, action-event combinations, temporal variables and a set of rules are used to represent constraint violations and their impacts on the simulated CM environment.

The limitation of this method was that event definitions could be used to explicitly identify defined temporal and resource constraints. However, implicit constraints that drive the schedule, but are not defined using the action-event combinations needed a separate reasoning system. For example, a dependency relationship between two unrelated activities that may result in a space conflict on site 
cannot be uniquely expressed using a typical action-event definition. It becomes more difficult to deterministically define $E_{\text {prec }}$ whenever a constraint violation can be arrived at through more than one sequence of occurrences. Hence, the next step in the development process established a simple temporal constraint network called TONAE to establish a variety of constraint relationships to describe the construction domain with the ability to express uncertainty and quantifiable consequences of constraint violations.

Simple Temporal Networks (STNs) (Dechter et al 1999) and their associated polynomial time algorithms are widely used in temporal reasoning. An STN includes nodes, representing time points, and edges connecting nodes, representing temporal constraints. The temporal constraints have a form such as $\left[2 \leq\left(\mathrm{x}_{2}-\mathrm{x}_{1}\right) \leq 5\right]$ meaning that the duration between time points $x_{1}$ and $x_{2}$ can be between 2 to 5 units of time. This allows the definition of hard constraints and deadlines between two time points, as well as the definition of temporal tolerances associated with a constraint. While the existing representation defined intervals only as activities with deterministic durations - STNs extend the representation to include intervals that represent constraints between one or more activities, and events with variable durations.

The STN representation of the CM domain is called TONAE (TempOral Network with Activities and Events) and it defines three classes of intervals that represent construction activities (relating the activity start time point and end time point), events (using a start time point and an end time point and relating each to the activities that are affected by the event) and constraints (relating start/end time points between activities and events). Each interval is defined by a duration upper bound and a duration lower bound indicating allowable temporal tolerances associated with the activities (float), constraints and events. In addition each interval is associated with a Cost Overrun Index (COI) that is indicative of the expenses/damages incurred when the duration of the specific interval exceeds the bounds. TONAEs advance the existing representation in three ways: 1.) They can represent intervals as well as constraints between individual time points defining such intervals, 2.) They provide an integrated approach to express all the constraints, activities and events using a temporal network and 3.) They provided the ability to develop general algorithms that could be used to traverse and query the network to aid decision-making and planning. A detailed discussion of the TONAE and its associated algorithms can be found in Anderson et al. (2007). The input to this model is an as-planned project schedule, with explicit identification of significant constraints that drive the schedule, external events likely to happen during the project and associated probabilities.

Mathematical Model: A model was developed to mathematically express the relationships between cost, schedule and productivity in the CM domain (Rojas and Mukherjee 2003). This model is based in the assumption that the construction domain consists of resources - labor, equipment and material - each of which are associated with costs, that can be summed across activities, and on a daily basis. The sum of costs across a project resource loaded schedule provides the estimated project direct costs when it is implemented on schedule. Constraint violations described in the previous sub-section can result in impacts on project performance. This model includes update functions that calculate remaining activity durations, forecast 
productivity and expected cost over runs and compare As-Planned and As-Built performance. The input to this model is an extensive resource loaded schedule.

\section{Situational Simulation Implementation}

In this section we describe the pragmatics of the GPF, including descriptions of how it has been developed to represent simulations of construction projects and how participants can interact with it to explore "what-if" scenarios. The general architecture of the GPF implementation can be classified into three separate logical models: Process Model, Product Model, and the Information Model (Rojas and Mukherjee 2003). We discuss the pragmatics of implementing the ICDMA - a situational simulation of a real life steel framed office building built in 2003 (Daccarett and Mrozowski 2005) using the GPF within this framework.

Project Context: The building has four stories, has 80,000 square feet of built area, and weighs approximately 400 tons of structural steel or about 10 pounds per square foot. Fabrication and erection cost $\$ 9$ per square foot. A total of 964 prefabricated structural steel members were used in the construction. The standard bay size in the building is 30 feet by 30 feet and there are 3 bays along the width and 7 bays along the length of the building.

Process Model: The process model consists of the Emulation Engine (EE). The EE is the fundamental underlying simulation engine and it implements the formal mathematical, representation and reasoning models discussed above. The granularity of the simulation in ICDMA is a week. Activity durations, and constraints are expressed using the TONAE. An example of a critical constraint is: the hoisting operation for a sequence of a higher storey has to start after a considerable portion of the decking operation for sequence of the immediately lower storey had been completed to afford safe fall distances. Given the numbering scheme of the sequences, this translated to a relationship between the mid-point of the Decking activity of sequence $i$, and the starting point of the hoisting activity of sequence $(i+2)$. This and other similar constraints were expressed using the TONAE Event information was encoded as follows: Moderate rain on site can result in a $50 \%$ loss of productivity, last for a duration of a week, with a probability of $10 \%$. The simulation also encoded events such as worker fatigue, labor strike and failed material delivery.

Information Model: The information model consists of all the relevant information that is necessary to simulate a specific construction project. While the EE define the machinery of the GPF, the information model includes schedule, cost and constraint information about specific construction projects that are simulated by the GPF. Specifically, the information used in ICDMA were:

- A resource-loaded schedule (32 weeks) representing the six sequences in which the steel framed structure was constructed. Each sequence consisted of hoisting, bolting and connection work and decking activities, and represented the order in which sections of the frame were erected.

- For each activity, the associated resource costs were also included. For example, each hoisting activity was associated with the specific steel members hoisted, the hoisting crew, the crane equipment used and the expected production rate of the activity. This was done for each activity in each of the six sequences.

- Information on events and constraints that were used to build the TONAE. 
The project specific information is stored in a database server, accessible by the EE.

Product Model: The product model consists of the Visualization Engine (VE). It implements an interactive interface to the EE allowing human participants to run the simulation of a construction project and make critical decisions. It visualizes information regarding schedule, cost and commodity curves (Figure 1) and material, labor and equipment resources available (Figure 1.) to the decision-maker at any time in the simulation. In addition it provides an interface that captures the decisions provided by the participant and relays it to the EE. Participant decisions include purchase, allocation and re-allocation of resources to activities (labor and material), changes in productivity related variables. All updates in schedule and cost calculated by the $\mathrm{EE}$ in response due to decisions, and current simulation information regarding events and constraint violations, are communicated to the participant through the VE.

System Architecture: The database server, EE and VE together define a webbased architecture of situational simulations. The EE and the database server reside on a central server, while the VE is a thin client that is deployed to the participant's desktop. Via the web, the client communicates with the server and functions as the product interface between the simulation and the human participant. The motivation behind this architecture is to ensure that all data is securely stored on the web-server and to collect decision-making data from the human participants during the simulation for future data mining purposes. In addition, it avoids transferring heavy simulation computing and processing to the client running the simulation, only requiring them to be connected to the Internet. ICDMA was implemented using Java 1.5 SDK and a PostgreSQL database server. This ensured that the simulation remains platform independent and easily accessible by diverse operating systems used by participants across academia and industry.

\section{Research Methods Using Situational Simulations}

In this section, we discuss current research and future directions of applying situational simulations to construction education, construction contingency planning, and the study of decision-making in dynamic environments.

Construction Education: Situational simulations are used in construction education to provide students clinical exercises in decision-making within interactive dynamic virtual environments. There is evidence in education literature that interactive virtual environment allow students to explore and construct knowledge through authentic experience, thus enhancing their ability to learn by connecting ideas that are otherwise provided through fragmented classroom discourse (Rojas and Mukherjee 2005b).

The Virtual Coach implementation was used to test the ability of situational simulations in teaching undergraduates using a hypothetical 12-activity project. 19 senior level CM students tested the simulation, with the goal of completing the project within budget and schedule in the face developing crisis scenarios. They were provided a simple pen-paper test on crisis management before and after the simulation intervention - using the pre-test/post-test protocol. The hypothesis of the test was: a statistically significant difference in performance on the test can be attributed to improved conceptual understanding due to participation in the simulation. In addition, qualitative feedback was collected from the students. The 
results showed that student test scores significantly improved with respect to managing precedence and temporal constraints driving the construction project and in their ability to identify and relate critical path to construction cost (Rojas and Mukherjee 2006).

Currently a vastly improved version of the Virtual Coach, using a complex real life construction project is being developed with close collaboration with construction companies. In addition, the interface is significantly being improved to display multi-modal construction information. In the course of the next academic year the system will be deployed and tested as an educational intervention across several participating partner schools in the US and Canada. This is a significant contribution of this research to construction education.

Construction Contingency Planning: Based on the formal models driving situational simulations, it can be used as a conformant planner - a system that can automatically create contingency plans in two ways: (i) accounting for typical contingent situations (such as weather events) and (ii) automatically estimating contingencies that are difficult to foresee, by exploring combinatorial future spaces resulting from constraint violations as expressed on the TONAE. The querying algorithm discussed in Anderson et al. (2007) can estimate and classify risks in dynamic construction scenarios as situations evolve and classify the associated risks by impact and probability by enumerating Monte Carlo samples of the combinatorial future paces. The approach was validated using ICDMA to classify risk in the construction of the steel framed building. The project involved three external events and 12 temporal and resource constraints with varying probabilities of occurrence (see project context and description above).

Traditional contingency estimation in construction primarily uses probabilistic approaches based on pre-defined risk scenarios with limited dynamic exploration of decision outcomes. This approach builds on existing methods, and further enhances them by including the ability to estimate contingencies from scenarios that can be simulated as combinations of possible constraint violations in a dynamic environment. This is a step toward the development of systems capable of automatically generating and classifying contingency scenarios during the planning phase of construction projects (Anderson et al. 2007).

Decision-Making in Dynamic Environments: Situational Simulations can also be used as experimental test beds for studying decision-making in dynamic, uncertain environments - specifically the impact of critical decisions in construction crisis scenarios. This research draws on methods in dynamic decision-making and construction data mining. The goal of this research is to use data mining approaches to classify decisions based on their project impacts. The hypothesis is that efficient decisions have structure that reflects underlying project constraints, and the relationship between how expert decision-makers organize and relate general domain knowledge to specific situation awareness. The data to be analyzed consists of decisions taken by construction managers with varying levels of expertise in fast evolving crisis scenarios. Given that it is very difficult to collect human decisionmaking data in controlled crisis scenarios - situational simulations of realistic construction projects provide the necessary experimental test beds to collect and analyze data. 


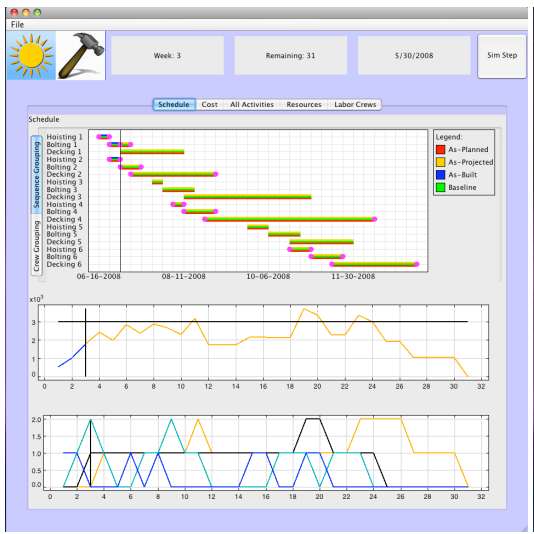

(a)

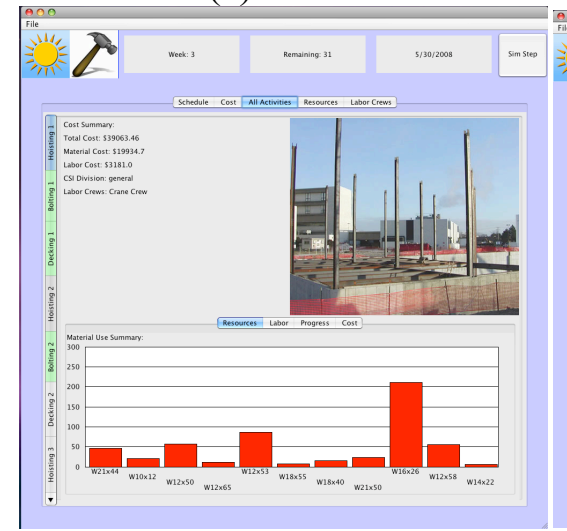

(c)

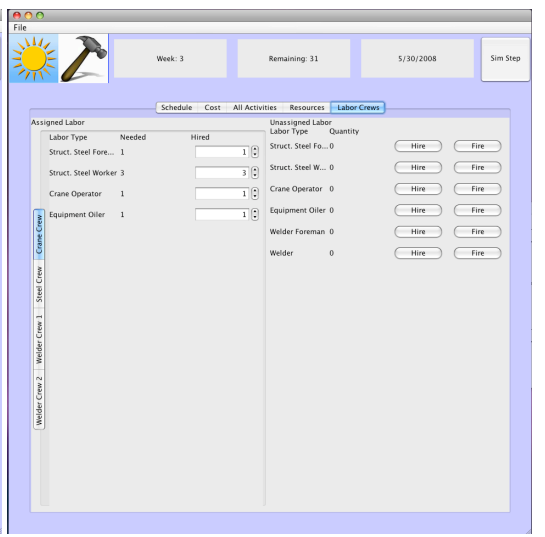

(b)

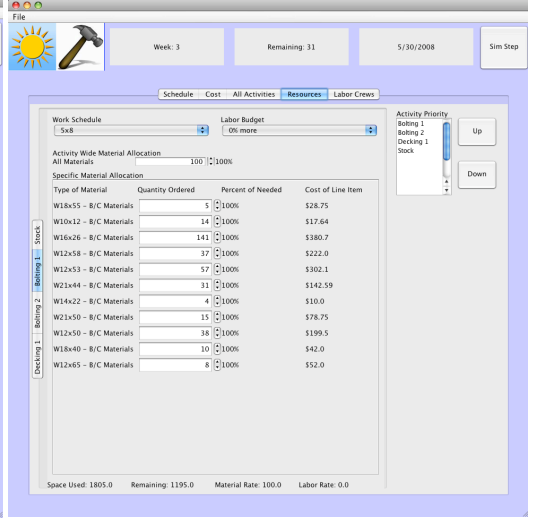

(d)

Figure 1. ICDMA: (a) Schedule and Commodity curves (b) Labor resources (c) Activity specific information (d) Material Resources

Current research (Watkins et al. 2008) addresses challenges in the collection, organization and analysis of human subject data using situational simulations such as the ICDMA. A formal data collection framework has been developed that can prescribe data collection protocols in a situational simulation and establishes mathematical approaches to organizing and classifying them. Preliminary results show that situational simulations can be used to successfully collect decision-making by controlling the simulation environment by situation and user particulars. In addition, such data can be used to mathematically construct decision shape vectors that can be used to quantify and classify decisions. Preliminary validation of the system has been conducted using participants from the industry and academia.

This research furthers our understanding of construction decision-making in particular and decision-making in dynamic uncertain crisis scenarios in general. Situational simulations can also be extended to study critical decision-making in fast evolving crisis scenarios involving multiple co-dependent infrastructure systems - a necessary and important area of investigation.

\section{Conclusion}

In this paper we have summarized the conceptual and formal models driving situational simulations, associated research methods, current results and theoretical contributions, and future directions in construction research and education. It is 
important to note that the interdisciplinary scope of this research involves diverse teams from engineering, construction management, computer science, education and the construction industry.

\section{Acknowledgement}

This research in this paper was developed under a grant from the NSF grant \#SES 0624118 to PI: Amlan Mukherjee, and Fund for the Improvement of Postsecondary Education (FIPSE), U.S. Department of Education, grant \#P116B060047 to PI: Eddy Rojas (University of Washington), Amlan Mukherjee (Michigan Tech). However, these contents do not necessarily represent the policy of the NSF or the Department of Education, and there should be no assumption of endorsement by the Federal Government.

\section{Reference}

Anderson, G. R., Onder, N., and Mukherjee, A. (2007). "Expecting the unexpected: representing, reasoning about, and assessing construction pro ject contingencies." Winter Simulation Conference, S. G. Henderson, B. Biller, M. H. Hsieh, J. Shortle, J. D. Tew, and R. R. Barton, eds. WSC, 2041-2050.

Daccarett, V., and Mrozowski, T. (2005) "Aisc digital library: Construction management of steel construction." Available via $<$ http://www.aisc.org/> accessed 01/10/2007].

Dechter, R., I. Meiri, and J. Pearl. (1991). "Temporal constraint networks." Artificial Intelligence 49:61-95.

Hajjar, D. and AbouRizk, S. (1999) "Simphony: an Environment for building Special Purpose Construction Simulation Tools" Proceedings of the Winter Simulation Conference, 1999, P.A.Farrington, H.B.Newbhard, D.T.Sturrock and G.W.Evans, eds.

Martinez, J. and Ioannou, P. (1999). "General-purpose systems for effective construction simulation," Journal of Construction Engineering and Management, 125 (4), 265-276.

Rojas, E., and Mukherjee, A. (2003) "Modeling the Construction Management Process to Support Situational Simulations." Journal of Computing in Civil Engineering, ASCE, 17(4) pp 273-280.

Rojas, E. and Mukherjee A. (2006) "A Multi-Agent Framework for General Purpose Situational Simulations in the Construction Management Domain," Accepted by the Journal of Computing in Civil Engineering, ASCE, 20(6) pp 1-12

Rojas, E. and Mukherjee A. (2005a) "Interval Temporal Logic in General Purpose Situational Simulations," Journal of Computing in Civil Engineering, ASCE, 19(1) pp 83-93

Rojas, E. and Mukherjee A. (2005b) "A General Purpose Situational Simulation Environment for Construction Education," Journal of Construction Engineering and Management, ASCE, 131(3) pp 319-329

Watkins, M.T., Mukherjee, A. and Onder. N. (2008) "Using situational simulations to collect and analyze dynamic construction management decisionmaking data." In Proceedings of the Winter Simulation Conference (ACM/SIGSIM), 2008 . 